\title{
Field strain measurement on the fiber-epoxy scale in CFRPs
}

\author{
Ran Tao ${ }^{\mathrm{a}}$, Ali Moussawi ${ }^{\mathrm{a}}$, Jian Zhou ${ }^{\mathrm{a}}$, Gilles Lubineau ${ }^{\mathrm{a}}$, Bing Pan ${ }^{\mathrm{b}}$

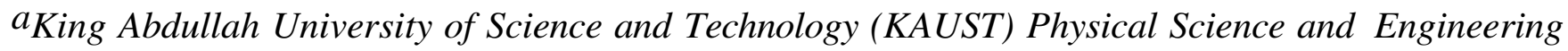 \\ Division. Cohmas laboratory \\ Thuwal, 23955-6900, Saudi Arabia Tel : (+966) (0) 28082983

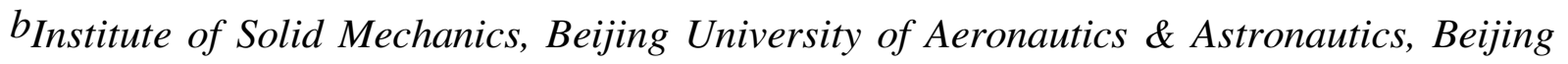 \\ 100191, China
}

\begin{abstract}
Laminated composites are materials with complex architecture made of continuous fibers (usually glass or carbon) embedded within a polymeric resin. The properties of the raw materials can vary from one point to another due to different local processing conditions or complex geometrical features for example. A first step towards the identification of these spatially varying material parameters is to image with precision the displacement fields in this complex microstructure when subjected to mechanical loading. Secondary electron images obtained by scanning electron microscopy (SEM) and then numerically deformed are post-processed by either local subset-based digital image correlation (DIC) or global finite-element based DIC to measure the displacement and strain fields at the fiber-matrix scale in a cross-ply composite. It is shown that when global DIC is applied with a conformal mesh, it can capture more accurate local variations in the strain fields as it takes into account the underlying microstructure. In comparison to subset DIC, global DIC is better suited for capturing gradients across the fiber-matrix interfaces.
\end{abstract}

Keywords: Numerical simulation, Image correlation, Electron microscopy, Distortion, Conformal mesh

\section{Introduction}

The structural properties of carbon fiber reinforced polymers (CFRPs) are strongly related to their micro-structure and micro-mechanical behavior. The local behaviors of both the matrix and the fiber-matrix interface play an important role in the way the degradation mechanisms (such as initiation and percolation of cracks) develop and spread [1]. When the material is subjected to controlled mechanical loading, high-accurate kinematic fields obtained from the full field measurement can be used to access the material constitutive relations [2,3]. The unique features of DIC (Digital Image Correlation) make it a very convenient and fast tool to track and measure such complex displacement fields at the low scale, opening new directions towards a better evaluation of mechanical properties of both the matrix and the fiber-matrix interface.

In the complete approach, Scanning Electron Microscopy (SEM) is used to record high-resolution images at different loading stages. These images are then numerically compared in order to evaluate the map of relative displacement fields between the different configurations [4]. Two main DIC techniques are available for image processing, local subset-based DIC [5] and global FE-based DIC [6]. In subset DIC, the region of interest ROI within the image is discretized into subsets which are independently correlated, the result being a displacement vector for each subset. Displacements between two subsets are a prior not related during the correlation process. On the other hand, global DIC uses finite elements to interpolate the displacement field over the whole ROI. The mesh may be built to consider the main micro-structural features to yield a better 
strain measurement. Most of the current work has been performed using subset DIC [7], but the intense gradient in the strain at the fiber-epoxy interface is greatly smoothened because of the inability of the subset-based approach to handle the strain discontinuity between dissimilar materials. A detailed comparison work between the subset-based DIC and the global FE-based DIC has been recently reported in [8].

Our objective in this paper is to better resolve the displacement and strain fields at the fiber-matrix scale, even close to the fiber-matrix interface. This is a first proof of concept in which the displacement is not experimentally generated, but real experimental reference images are first deformed numerically. FE-based global image correlation, where the FE mesh is conformal to the underlying micro-structure heterogeneity, is applied to numerically deformed SEM speckle images at the fiber matrix scale. The global FE-based DIC will be compared with local subset-based DIC to demonstrate whether it can better capture sharp gradients in the kinematic fields across interfaces. The sources of error in the displacement fields obtained from SEM images will be analyzed, in order to give a more practical road to the actual experimental implementation.

A short introduction of DIC which highlights the differences between the subset and FE-based techniques (Sec. 2) follows this introduction. Then, the procedures for specimen preparations in order to obtain actual SEM speckle images are detailed in Sec. 3, the sources and correction principles of distortion in SEM imaging is discussed in Sec. 4, and finally the implemented results of the strain measurement of numerically deformed SEM speckle images by the global FE-based DIC, and the comparison of the subset-based DIC and the global FE-based DIC are demonstrated in Sec. 5.

2.

Subset and global correlation

DIC is an effective and practical technique to achieve the full-field measurement. DIC has the advantages of scalability and non-intrusiveness. Two main techniques are applied in the DIC algorithm, one is the subset DIC and the other one is the global FE-DIC. A short review of these two methods is presented in this section.

In the subset DIC, a square subset window centered at the point $\mathrm{P}\left(x_{0}, y_{0}\right)$ in the reference image is correlated to find a corresponding area in the deformed image [4]. The displacement mapping function (for example, Eq. 1) is usually assumed to represent the uniform subset deformation, where $\mathrm{Q}(x, y)$ is a position in the reference subset, and $\mathrm{Q}^{\prime}(\bar{x}, \bar{y})$ is the corresponding position in the deformed subset, as showed in Fig. 1 a). This independent correlation algorithm will result in non-compatibility on the boundary of adjacent subsets.

$$
\left\{\begin{array}{l}
\bar{x}=x+u+\frac{\partial u}{\partial x_{x_{0}, y_{0}}}\left(x-x_{0}\right)+\frac{\partial u}{\partial y_{x_{0}, y_{0}}} \quad\left(y-y_{0}\right) \\
\bar{y}=y+v+\frac{\partial v}{\partial x_{x_{0}, y_{0}}}\left(x-x_{0}\right)+\frac{\partial v}{\partial y_{x_{0}, y_{0}}} \quad\left(y-y_{0}\right)
\end{array}\right.
$$

In the global FE-DIC, the whole ROI is discretized into finite elements linked by nodes. A set of global interpolation functions $N_{i}(i=1,2, \cdots, m)$ are applied to the nodal displacement $\left(\widehat{u}_{l}, \widehat{v}_{l}\right)(i=1,2, \cdots, m)$ [6]. Given that $(x, y)$ is an arbitrary point in the reference image, and $(\bar{x}, \bar{y})$ represents the corresponding point in the deformed image, Eq. 2 gives the displacement mapping between the reference and the deformed images. The compatibility of the displacement field is satisfied since all nodal displacements are correlated dependently. The advantages of the global method using conformal mesh is the ability to capture the large strain gradient at the dissimilar material interface.

$$
\left\{\begin{array}{l}
\bar{x}=x+u=x+\sum_{i=1}^{m} N_{i} \widehat{u_{i}} \\
\bar{y}=y+v=y+\sum_{i=1}^{m} N_{i} \widehat{v_{i}}
\end{array}\right.
$$


a).

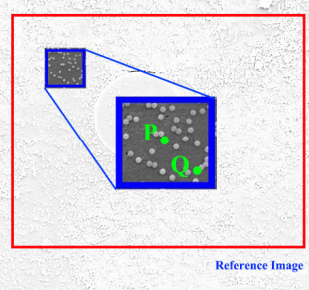

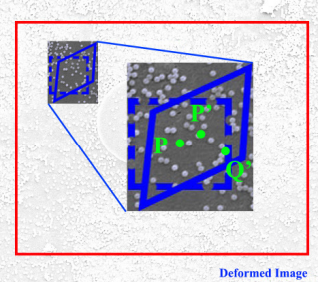

Deformed Image b).

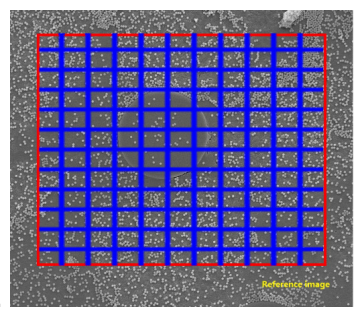

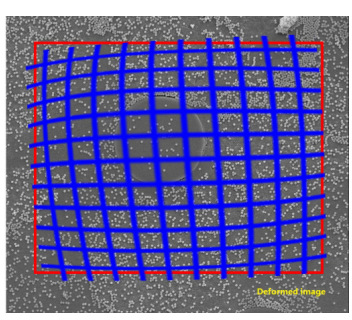

Fig. 1 Schematic figures showing: a).the correlation procedure using subset-based DIC, b). the correlation procedure using global FE-based DIC

\section{3.}

\section{Specimen preparation}

Actual speckle images acquired under the SEM will be post-processed by both subset DIC and global FE-DIC.

The $\left[0_{2} / 90_{10}\right]_{s}$ cross-ply plate is made by stacking prepregs $(300 \mathrm{~mm} \times 300 \mathrm{~mm})$ provided by HexPly ${ }^{\circledR} \mathrm{M} 21$. This $6 \mathrm{~mm}$ thick plate is made to laterally suit in the tensile machine, to be observed in the transverse direction, Fig. 2 a).

In order to view the distribution of fibers under the SEM, the sample is cut into small specimens with a dimension of $60 \mathrm{~mm} \times$ $6 \mathrm{~mm} \times 2 \mathrm{~mm}$ (length $\times$ width $\times$ thickness). The sanding process is conducted following an order of 320-500-1000-2400- 4000 and a sequential 3 microns- 1 micron polishing process. The root mean square of surface roughness $R_{q}=0.32 \mu m$ is obtained by a Zygo profiler after sanding and polishing, which indicates the surface smoothness limit of the as-prepared specimens.

Specimens are carefully imprinted with a nanoparticle-based speckle pattern for the DIC implement. At first, a 5-minute plasma surface treatment is applied to increase the hydrophilicity. A deionized water solution with a concentration of $1 \%$ w/v of PS (polystyrene) nanoparticles (diameter $173 \mathrm{~nm}$ ) is spin coated to have a uniform nanoparticle distribution on the specimen surface.

a).

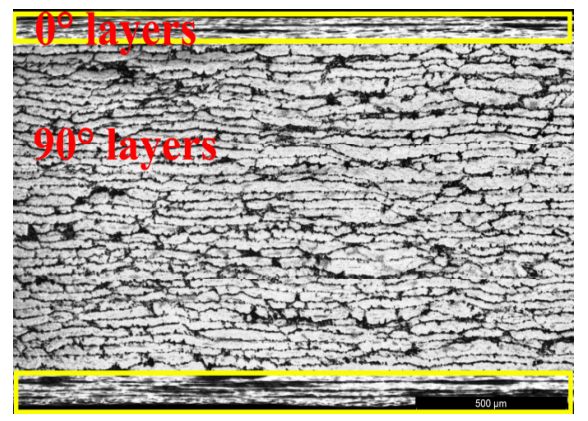

b).

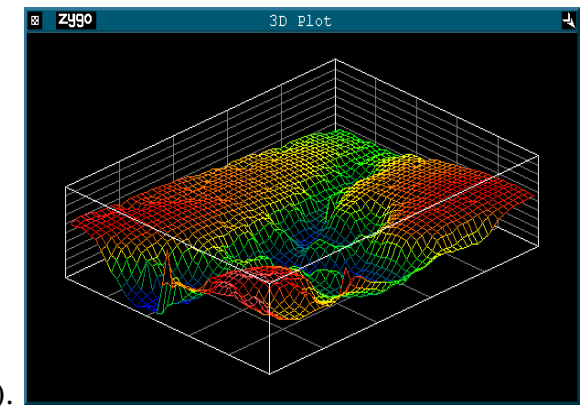

c).

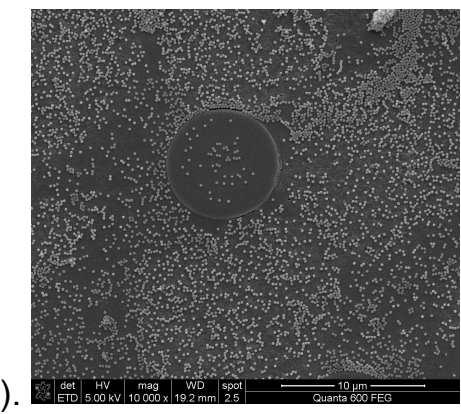

Fig. 2 a). 24-layer specimen observed laterally in the transverse direction. b). 3D surface profile from the Zygo profiler: the higher red region shows the fiber rich region, while the blue region is the matrix rich region. c). Speckle image with 173nm PS nanoparticles under SEM at the 10,000 magnification

\section{4.}

\section{SEM imaging distortion}

In the following, the surface fiber distribution is observed under SEM via the secondary electron (SE) detector with an accelerating voltage of $5 \mathrm{kV}$ and a spot probe size of 2.5 .

SEM imaging can be polluted by specific error sources such as drift that would not appear in classical optical imaging. In the SEM, an electron beam probes one position at each time, and the emerging signals of that position are collected by the SE detector. In this rasterization scanning, different motion could happens between pixels images at different time, which is known as drift distortion [9]. The scanning behavior of the SEM is shown as straight lines with arrows in Fig. 3, where the blue frame represents the acquired image.

Besides, the scanning positions and the signals collection in the SEM are controlled by voltage, leading to a more complex spatial distortion compared to optical systems. A nonparametric surface fitting method, rather than the parametric method commonly applied in the lens distortion, has to apply to correct the spatial distortion in the SEM $[9,10]$. 


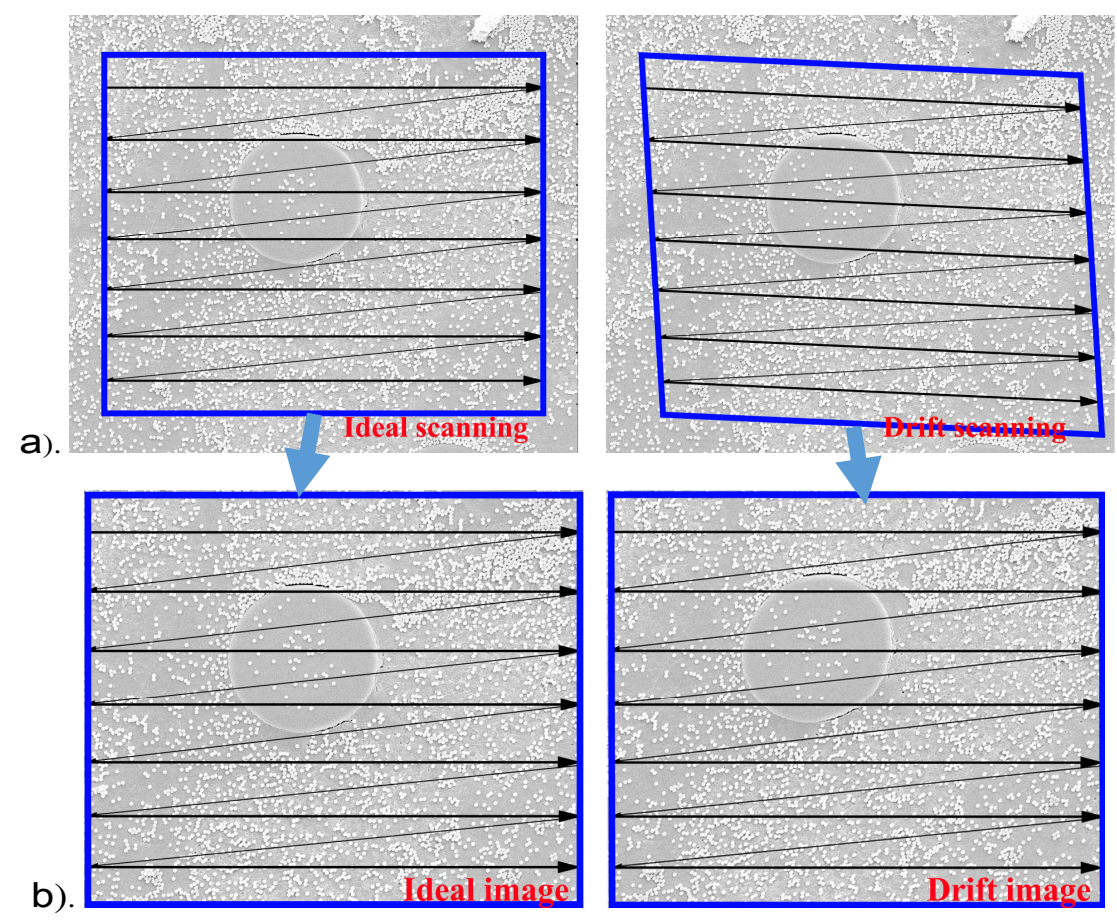

Fig. 3 Schematic figures showing: a). the ideal scanning and the drift scanning, b). acquired ideal and drift images via the ideal scanning and the drift scanning, respectively. The blue frame is the image plane, and black arrows demonstrate the scanning process of the electron beam

The principle procedure of correcting both the drift and spatial distortion is listed in the reference [9, 11], as shown in Fig. 4. This calibration procedure consists of 9 steps, and two images are acquired at each step to extract drift displacements. The drift distortion is corrected at first. The drift correction gives 9 drift-free images, and then the spatial distortion correction is applied to these remaining 9 images.

In this research, distorted images are simulated based on actual drift parameters, artificial spatial functions and SEM speckle pattern images. The effectiveness of the distortion correction is demonstrated through numerical calibration procedure.

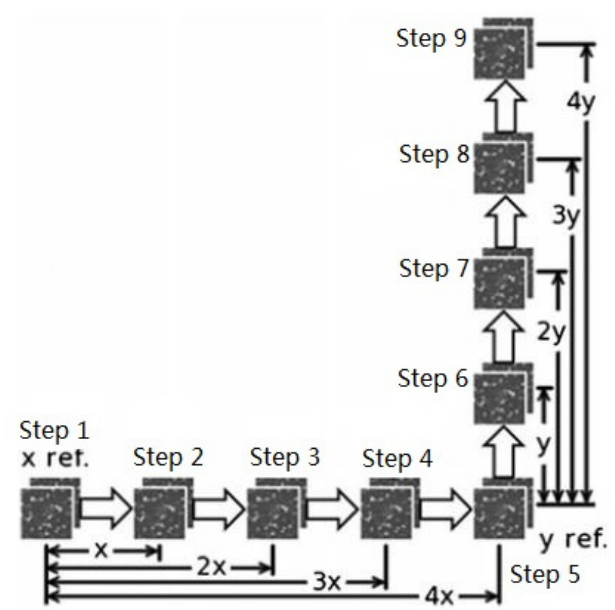

Fig. 4 A Schematic illustration of the calibration procedure $[9,11]$

\section{5. $\quad$ Numerical examples}

Actual speckle images acquired under SEM with random and uniform speckles on the isolated fiber region are selected as the reference images, which are deformed numerically in the following simulation examples. Validation of the drift distortion 
method is demonstrated at first, and then followed by the discussion about the global FE-DIC implementation.

\subsection{Drift correction}

Simulated drift images are numerically deformed from one actual SEM speckle image with an artificial drift velocity. Drift displacements derived from the correction method are compared to prescribed artificial displacements to evaluate the quality of the correction procedure.

To capture the drift behavior on the Quanta 600 SEM, the speckled specimen, which was clamped in the micro-tensile machine to present a same configuration as the actual experiment, was observed under SEM. 60 images were obtained within 2 hours without any specimen movement at three different magnifications. Image sequences are analyzed by a commercial subset DIC software Vic2D and the average frame displacements against the ending time of each scan is plotted in Fig. 5. An average drift velocity was found to be 0.2 pixels per minute (both in $\mathrm{x}$ and $\mathrm{y}$ direction) at 10,000 magnification. It is derived by dividing the final displacement by the total time period.

a).

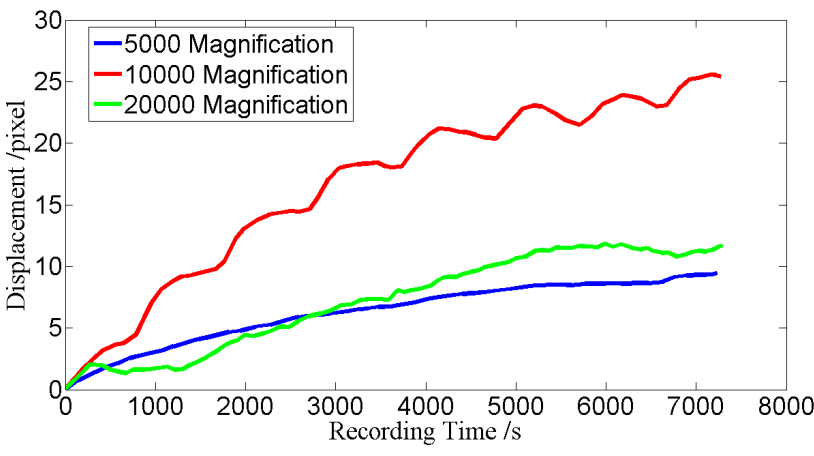

b).

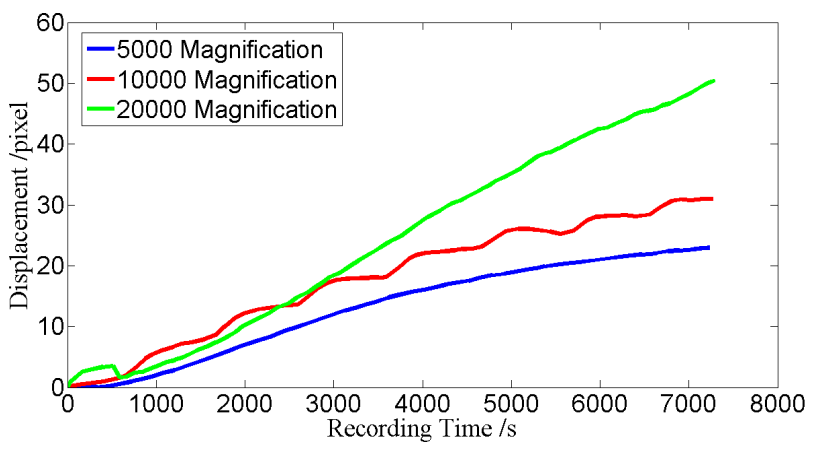

Fig. 5. Average SEM drift displacements versus the ending time of each scan: a). in $x$ direction (horizontal), b). in $y$ direction (vertical)

The prescribed drift velocity in the numerical simulation is chosen to be 0.2 pixels per minute, to be representative of the actual drift distortion under SEM at 10,000 magnification. As described above, the calibration procedure consists of 9 pairs of images. The drift correction method gives the drift displacement of the first image in each pair, which is compared to the prescribed drift displacement. The average correction error is represented by the difference between output displacements from the correction and prescribed drift displacements, which are listed in Table 1. Since the first image of the first step is set as the reference with a zero input drift displacement and zero corrected output displacement, the error is zero demonstrated in Table 1.

Table 1. Drift Correction Error

\begin{tabular}{|c|c|c|c|c|c|c|c|c|c|}
\hline Step No. & 1 & 2 & 3 & 4 & 5 & 6 & 7 & 8 & 9 \\
\hline Error (pixel) & 0 & -0.0402 & -0.0278 & -0.0685 & -0.1610 & -0.1455 & -0.1828 & -0.2683 & -0.2584 \\
\hline Error $(\%)$ & 0 & -6.24 & 1.94 & -0.39 & -4.01 & -0.84 & -1.69 & -4.56 & -2.84 \\
\hline
\end{tabular}

\subsection{Spatial correction}

The spatial distortion is numerically simulated by applying an artificial distortion to each image. Such spatial distortion could be corrected by using the distortion correction module of the commercial subset DIC software Vic2D

Artificially prescribed spatial distortion functions are shown in Fig. 6, which have very different shapes from the conventional optical distortion function. The standard deviation of displacement fields before and after the distortion correction module is demonstrated in Fig. 7. Since only rigid body translation is performed during the calibration procedure, the spatial distortion is represented by large standard deviation values. The dramatically drop of standard deviation values demonstrate a successfully correction on the spatial distortion. 

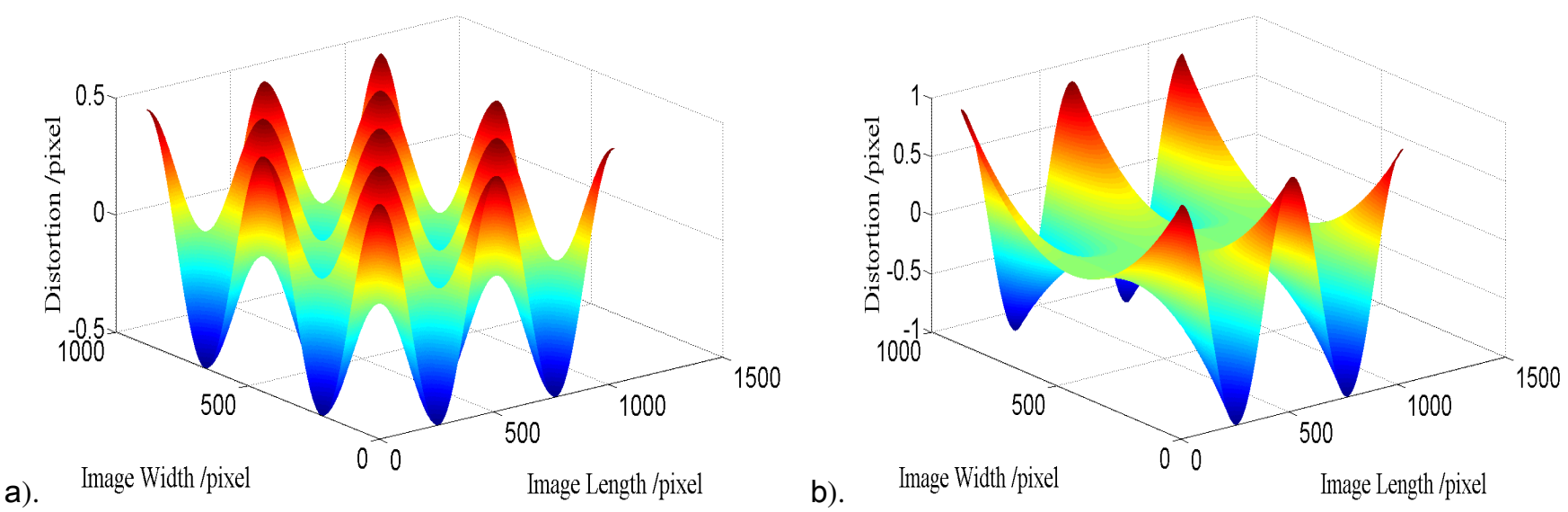

Fig. 6. Artificial spatial distortion functions: a), in $\mathrm{x}$ direction (horizontal), b). in y direction (vertical)
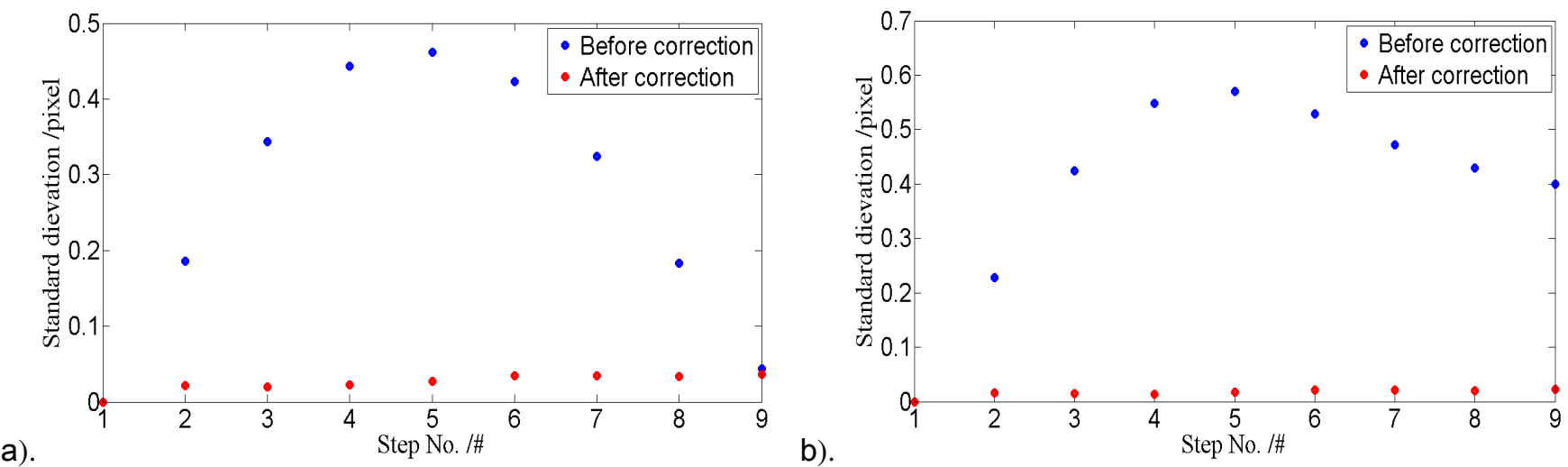

Fig. 7. Standard deviation of spatial displacements in different calibration steps: a). in $x$ direction (horizontal), and b). in y direction (vertical)

\subsection{Global DIC Implementation}

The global FE-DIC requires three input files: two speckle images of different configurations, an initial guess of the displacement field and the nodal data of the conformal mesh. The procedure flow is showed in Fig. 8.

A uniaxial deformation is simulated to an isolate fiber region containing only two circular carbon fibers, and it is solved with a highly refined mesh in ABAQUS. The displacement field from ABAQUS is used to numerically deform the reference speckle image, while the strain field will be used to compare with the subset DIC and the global FE-DIC output displacement fields.

Because of the non-linear feature of the global FE-DIC, the Newton-Raphson method is applied in the algorithm. It is very vital to choose a good initial guess, to have faster and more accurate iteration results. During this simulation, the commercial subset DIC software Vic2D is applied to obtain initial displacement fields, which are interpolated to give initial nodal displacements.

The conformal mesh is essential to better capture the sharp strain gradient at the fiber-matrix interface. Since the preknowledge of the distribution of different materials is easily obtained from high magnification SEM images, conformal mesh is able to well capture the geometry, reducing interpolation errors across the interface. Node information of conformal quadratic elements is extracted from ABAQUS and then input into the global FE-DIC algorithm. 


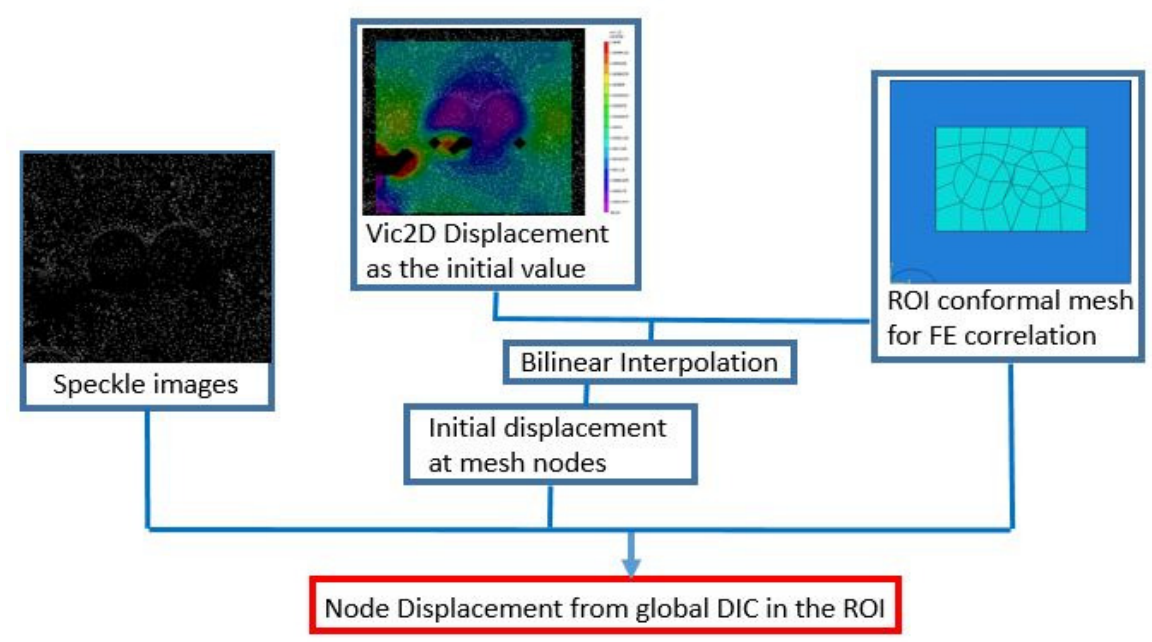

Fig. 8 A Schematic figure of the implementation procedure of global FE-based DIC

After having obtained the nodal displacements from the global FE-DIC method, the displacement field is obtained from the interpolation of finite element shape functions. The displacement field may be noisy because of speckle, mesh and image noise, which could result in larger error when deriving strain field. The SG (Savitzky-Golay) filter is applied to reduce the displacement noise, improving the strain accuracy. However, a large strain window will introduce large smoothen effect, and decrease the quality of strain field especially at the interface. The size of the strain window is carefully chosen to have more accurate strain with less smoothen effect.

A strain window with a size of 19 pixels is chosen in this simulation configuration. The strain field $E_{x x}$ is shown in Fig. 9, with a high strain between two fibers, which is compliant with the FEM strain field. A strain comparison plot along a horizontal line, combined with speckle and mesh information, is also shown in Fig. 9. It is obvious that the green line (global FE-DIC data after SG filter) shows better properties at the fiber-matrix interface compared to the red line (subset DIC data from Vic2D).
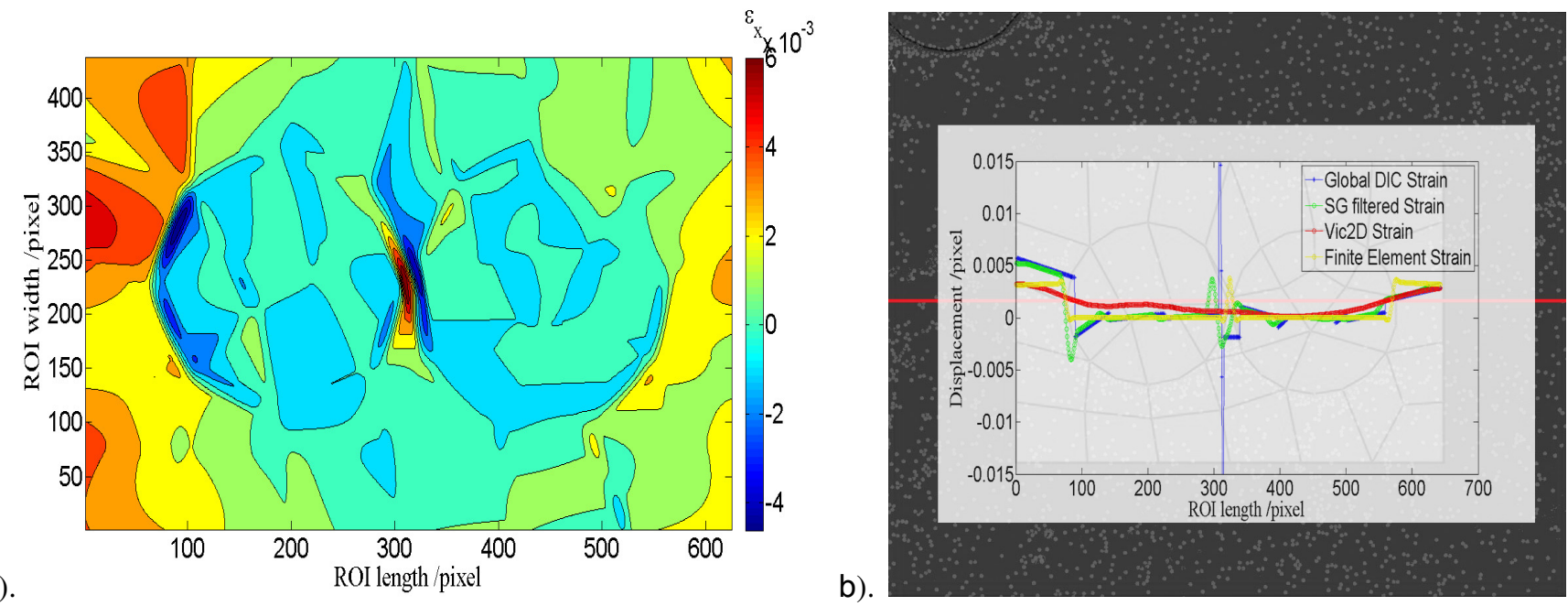

Fig. 9 The global FE-DIC strain field results with the strain window with a size 19 pixels. a). The strain contour of the $E_{x x}$ strain field. b).The strain field comparison along a horizontal line combined with the speckle and mesh information between: the direct global FE-DIC result (blue), the global FE-DIC result after the SG filter (green), the subset DIC Vic2D result (red) and the FEM result (yellow)

6.

\section{Conclusion}

The drift correction method and a global FE-DIC algorithm with a conformal mesh on numerically deformed SEM speckle 
images are implemented in this work. The effectiveness of the drift correction method is demonstrated by numerical experiments using simulated drift SEM speckle images. The global FE-DIC, where conformal quadratic elements are assembled through the commercial software ABAQUS, has the ability to better capture the large gradient at the fiber-epoxy interface. The strain fields from subset DIC, the global FE-DIC and the FEM along a horizontal line across the ROI are compared, and the global FE-DIC shows better consistency with FEM at the fiber-matrix interface.

In conclusion, the global FE-DIC using a conformal mesh could improve the ability to capture strain fields at the fibermatrix interface compared to the subset DIC. Following this numerical validation and analysis, actual in situ SEM tensile/compressive experiments will be conducted and acquired images will be post-processed by both, the distortion correction method, and the global FE-DIC, to obtain more accurate displacement and strain fields within actual CFRPs under actual experiments.

[1] Llorca, J., González, C., J. M. Molina-Aldareguía, J. Segurado, R. Seltzer, F. Sket, M. Rodníguez, S. Sádaba, R. Muñoz, L. P. Canal, Multiscale modeling of composite materials: a roadmap towards virtual testing., Adv. Mater. (Deerfield Beach, Fla.) 23, 5130-47. 2011

[2] G. Lubineau, A. Moussawi, J. Xu and R. Gras. A domain decomposition approach for digital image correlation based identification of local elastic parameters. International Journal of Solids and Structures. v. 55, pp. 44-57. 2015

[3] E. Florentin and G. Lubineau. Identification of the parameters of an elastic material model using the Constitutive Equation Gap Method. Computational Mechanics, v. 46(4), pp. 521-531. 2010

[4] M. Grediac, The use of full-field measurement methods in composite material characterization: interest and limitations, Compos. Part A-Appl. S. 35, 751-761. 2004

[5] B. Pan, K. Li, W. Tong, Fast, robust and accurate digital image correlation calculation without redundant computations, Exp. Mech. 53, 1277-1289. 2013

[6] Y. Sun, J. H. L. Pang, C. K. Wong, F. Su, Finite element formulation for a digital image correlation method 44, 73577363. 2005

[7] L. Canal, C. González, J. Molina-Aldareguía, J. Segurado, J. LLorca, Application of digital image correlation at the microscale in fiber-reinforced composites, Composites Part A: Applied Science and Manufacturing 43, 1630-1638. 2012

[8] Pan, B., Wang, B., Lubineau, G., \& Moussawi, A. Comparison of Subset-Based Local and Finite Element-Based Global Digital Image Correlation. Experimental Mechanics, 1-15. 2015

[9] M. A. Sutton, N. Li, D. Garcia, N. Cornille, J. J. Orteu, S. R. McNeill, H. W. Schreier, X. Li, Metrology in a scanning electron microscope: theoretical developments and experimental validation, Measurement Science and Technology 17,2613-2622. 2006

[10] H. W. Schreier, Calibrated Sensor and Method for Calibrating Same, Patent Pending 1. 2006

[11] A. D. Kammers, S. Daly, Digital Image Correlation under Scanning Electron Microscopy: Methodology and Validation, Exp. Mech. 53, 1743-1761. 2013 